\title{
Über die Hautvenen des Fusses bei den Affen.
}

\author{
Von
}

\author{
Seiho Nishi. \\ (Anat. Inst., Tokyo Kaiserliche Universität.)
}

In meiner vorigen Abhandlung habe ich über die Venen der Affenhand bei einigen Katarrhinen- und Platyrrhinenarten bereits Aufschluss gegeben ${ }^{1}$. Im folgenden beschreibe ich meine Befunde über die Hautvenen des Affenfusses, welche ich an 19 Fusspaaren von Macacus, und zwar 15 von M. rhesus, 2 von M. mulattus, je 1 von M. cyclopsis, M. cynomolgus und M. lacép. und an je 1 Fusspaar von Semnopithecus entellus unter den Katarrhinen sowie von Hapale jacchus, Mystax rosalia, Lagothrix lagotrica und Cebus capucinus unter den Platyrrhinen untersuchen konnte ${ }^{2)}$.

Über das Venensystem des Affen sind unsere Kenntnisse überhaupt noch sehr mangelhaft; eine einzige systematische Untersuchung finden wir bei Hirota ${ }^{3}$, der das ganze Venensystem des Affen an 6 Exemplaren von Macacus fuscatus, je 2 von $M$. rhesus und Cercopithecus spec. und an einem Orang-Utan-Exemplar eingehend studierte. Seine Beschreibung der Venen des Fusses, soweit sie sich auf meine Arbeit bezieht, lautet kurzızusammengefasst wie folgt:-

1) Die Vv. digitales dorsales verlaufen den beiden Rändern der Rückenfläche jeder Zehe entlang proximalwärts und vereinigen sich zwischen den Zehen mit den gleichnamigen Venen der benachbarten Zehen zu Vv. digitales dorsales communes. Die V. digitalis dorsalis tibialis

1) Folia anatomica japonica 13, 1935.

2) Ein Teil des Materials wurde durch wohlwollende Unterstützung der HattoriHôkôkwai mir zur Verfügung gestellt, der ich hiermit meinen verbindlichsten Dank ausspreche.

3) Kyoto Igakkwai Zasshi 24, 1924. 
der Grosszehe mündet direkt in den Arcus venosus dorsalis (s. u.) und die $\mathrm{V}$. digitalis dorsalis fibularis der Kleinzehe in die V. digitalis dorsalis communis IV. ein.

2) Die Vv. intercapitulares verbinden die Vv. digitales dorsales mit den Vv. digitales plantares (profundae) sowie mit dem Rete venosum plantare.

3) Die Vv. digitales communes, 4 in Zahl, verlaufen über den Fussrücken proximalwärts und fliessen in den Arcus venosus dorsalis ein. Sie zeigen individuell stark variierende Anordnung.

4) Der Arcus venosus dorsalis liegt auf den Basen der 3 ersten Metatarsalia und bildet zwischen dem 1. und 2. Metatarsale einen Winkel, wo er die V. digitalis communis I. aufnimmt. Der tibiale Schenkel des Bogens (Radix tibialis der V. saphena parva) verläuft proximal-tibialwärts, steigt um das distale Ende des Malleolus tibiae herum (niemals vor demselben) an der dorsalen Seite des Unterschenkels proximalwärts, um hier in verschiedener Höhe in die $V$. saphena parva einzumünden. Der fibulare Schenkel (Radix fibularis der V. saphena parva) verläuft dagegen auf dem Fussrücken proximal-fibularwärts und wendet sich um das distale Ende des Malleolus fibulae herum proximalwärts, um sich an der dorsalen "Seite des Unterschenkels in die V. saphena parva fortzusetzen.

5) Das Rete venosum dorsale pedis ist hauptsächlich aus dem Arcus venosus dorsalis und den Vv. digitales communes zusammengesetzt.

6) Das Rete venosum plantare ist ein feinmaschiges Venennetz, aus dem die Venenäste beiderseits in die beiden Saphenawurzeln einfliessen.

7) Die V. saphena parva ist der einzige Venenstamm im Unterschenkel; die V. saphena magna ist erst beim Orang-Utan typisch ausgebildet.

Dies ist kurz zusammengefasst der Inhalt der Beschreibung von Hirota.

Da die Fussvenen bei den von mir untersuchten Macacus-Arten ausser individuellen Abweichungen keine Artspezifität aufzuweisen scheinen, während sie bei meinem Semnopithecus-Exemplar den Platyrrhinen-Typus zeigen, so fange ich zunächst mit den Macacus-Befunden an.

Auf der Rückenseite jeder Zehe ist wie am Finger gewöhnlich nur eine Vene auf einer Seite stark ausgebildet, V. digitalis dorsalis, welche an der Gross- und Kleinzehe die V. tibialis hallucis bzw. V. fibularis digiti V. darstellt. An der 2. Zehe liegt sie in der tibialen Seite, an der 4. dagegen in der fibularen Seite des Zehenrückens, während die homologe Vene in der entgegengesetzten Seite fehlt oder nur schwach entwickelt ist. An der 3. Zehe liegt die Vene an der tibialen Seite; in manchen 
Fällen verläuft eine Vene auch an der fibularen Seite, welche eventuell der einzige Venenstamm auf der Rückenseite der 3. Zehe sein kann ${ }^{1)}$. Jede V. digitalis dorsalis nimmt von der plantaren Seite der betreffenden Zehe je eine etwas stärkere V. digitalis plantaris (V. intercapitularis, Hirota; könnte auch V. interdigitalis genannt werden) über die betreffende Seite der Grundphalanx auf und setzt sich proximalwärts in die V. digitalis communis fort.

Auf dem Fussrücken kommen in typisch ausgebildeten Fällen im gauzen 6 Venenstämmchen: V. marginalis tibialis, Vv. digitales com-

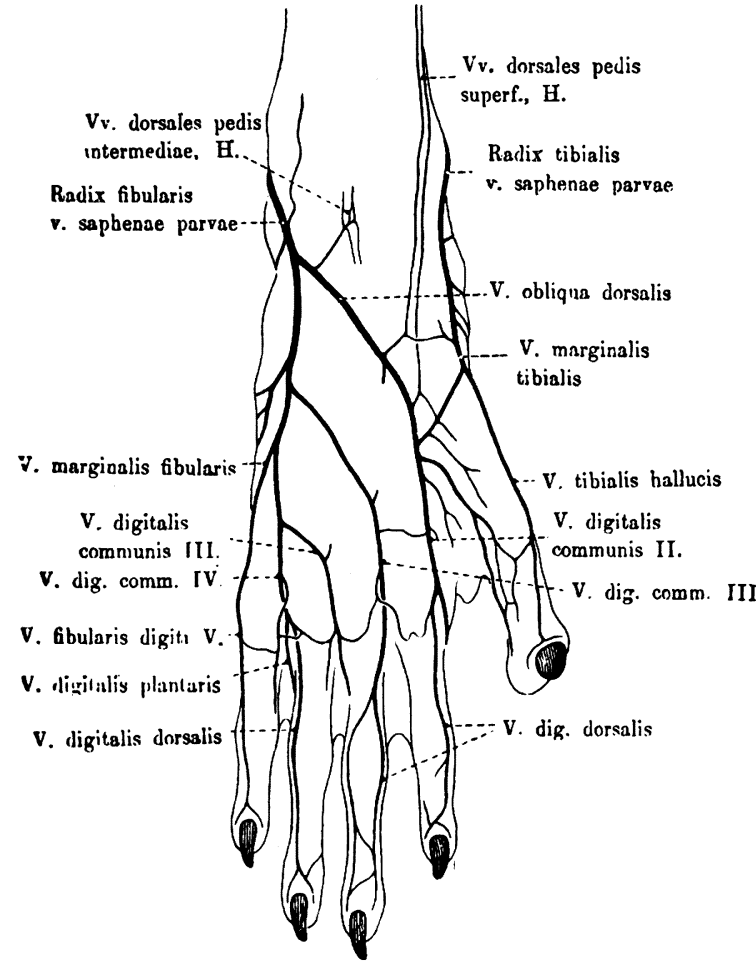

Fig. 1 .

Die Hautvenen am Fussrücken von Macacus rhesus. munes I.-IV. und V. marginalis fibularis. Die Marginalis tibialis (Radix tibialis der Saphena parva, Hirota) stellt die direkte proximale Fortsetzung der Tibialis hallucis dar, verläuft dem tibialen Rand des Fussrückens entlang proximalwärts und steigt über die tibiale Fläche des Malleolus tibiae hinweg in die dorsale Fläche des Unterschenkels weiter proximalwärts, um hier in verschiedener Höhe in die Saphena parva einzumünden. In ihrem Verlauf nimmt die Vene mehrere Venen vom Rete venosum plantare über den tibialen Fussrand auf. Die Marginalis fibularis entsteht dagegen aus der proximalen Fortsetzung der Fibularis digiti V., verläuft dem fibularen Rand des Fussrückens entlang proximalwärts und vereinigt sich vor dem Malleolus fibulae mit dem fibularen Schenkel des

1) Da die Injektion der Zehenvenen in vielen Fällen nicht genügend ausgeführt wurde, müssen die Befunde an der 3. Zehe noch durch weitere Untersuchungen bestätigt werden. 
Arcus venosus, dorsalis (s. u.). Der so gebildete Venenstamm (Radix fibularis der Saphena parva, Hirota) verläuft um den Malleolus fibulae weiter proximalwärts, um sich direkt in die Saphena parva fortzusetzen. Die Marginalis fibularis empfängt in ihrem Verlauf einerseits mehrere Venen vom Rete venosum plantare über den fibularen Fussrand und nimmt andererseits gewöhnlich, aber nicht immer, eine oder meherere Digitales communes auf ${ }^{1}$.

Die Digitales communes, 4 in Zahl, sammeln die Venen hauptsächlich von den 3 mittleren Zehen, und zwar die I. von der 2. Zehe, die II. von der 3. und die IV. von der 4., während die III., gewöhnlich schwächer ausgebildet, Venen von den entgegengesetzten Seiten der 3. und 4. Zehe empfängt. Ihre Anordnung auf dem Fussrücken ist individuell stark variierend, sodass eine Typeneinteilung kaum möglich erscheint. Die Digitalis communis I. teilt sich gewöhnlich bald in 2 Schenkel, von denen der tibiale über das Metatarsale I. hinweg schräg proximal-tibialwärts verläuft und mit der Tibialis hallucis gemeinsam die Marginalis tibialis bildet, während der fibulare Schenkel, V. obliqua dorsalis, über den Fussrücken schräg proximal-fibularwärts aufsteigt, um zusammen mit der Marginalis fibularis den Anfangsteil der Saphena parva zu bilden. In dieser Weise entsteht der Arcus dorsalis pedis, Hirota. Der tibiale Schenkel ist aber keineswegs ein konstantes Gebilde; er stellt manchmal nur eine schwache Anastomose zwischen der Marginalis tibialis und der Digitalis communis I. bzw. der Obliqua dorsalis dar, feblte auch tatsächlich in 4 Fällen, in welchen Fällen die Digitalis communis I. sich direkt in die Obliqua dorsalis fortsetzte. In anderen 5 Fällen fand die Verbindung in entgegengesetzter Richtung statt, sodass die Tibialis hallucis mit ihrem Hauptteil in die Obliqua dorsalis einlief. Der fibulare Schenkel, Obliqua dorsalis, ist dagegen viel ständiger. Nur in 2 Fällen fehlte sie und der Hauptstrom der Digitalis communis I. ergoss sich durch den tibialen Schenkel in die Marginalis tibialis.

Die Digitalis communis II. mündet entweder selbständig oder mit der III. oder mit der III. und IV. gemeinsam in die Obliqua dorsalis oder in die Marginalis fibularis ein. Am häufigsten (13 mal) mündete sie nach Aufnahme der III. und IV. in die Marginalis fibularis, ungefähr ebenso häufig (12 mal) selbständig in die Obliqua ein. Im ersteren Falle entsteht auf dem Fussrücken eine zweite schräge Vene, Obliqua dorsalis

1) Hirota nimmt die Digitalis communis als fibularen Venenstamm des Fussrückens an, in den die Fibularis digiti V. einmündet. In 4 Fällen fand ich indessen die Marginalis fibularis als direkte selbständige Fortsetzung der Fibularis digiti V., die also nicht von der Digitalis communis IV. aufgenommen wurde. 
distalis, welche ich unter 38 Fällen in 18 finden konnte. In 2 Fällen teilte sich die Digitalis communis II. bald in 2 Schenkel, von denen der fibulare die Obliqua distalis darstellte, während der tibiale Schenkel einmal in die Digitalis communis I., ein anderes Mal in die Obliqua proximalis einmündete. In einem Fall endlich fehlte die Vene und das tibiale Ende der Obliqua distalis vereinigte sich mit der tiefliegenden Metatarsea dorsalis III.

Die Digitalis communis III. ist, wie gesagt, gewöhnlich schwach ausgebildet. Sie entsteht aus den Venenstämmchen, welche von den entgegengesetzten Seiten der 3. und 4. Zehe kommen, und fliesst gewöhnlich entweder in die II. (18 Fälle) oder etwas seltener (13) in die IV. ein. Nur einmal mündete sie direkt in die Obliqua proximalis, 2 mal ebenfalls direkt in die Marginalis fibularis und 2 mal endlich in 2 Schenkel geteilt in die Digitalis communis II. und IV. ein.

Die Digitalis communis IV. mündete einmal selbständig und einmal nach Aufnahme der III. in die Obliqua proximalis, 10 mal selbständig in die Obliqua distalis und $8 \mathrm{mal}$ in die Marginalis fibularis, 3 mal nach Aufnahme der III. in die Obliqua distalis und $9 \mathrm{mal}$ in die Marginalis fibularis ein.

Hirota unterscheidet bei seiner Einteilung 5 Typen, unter welchen 2 Typen am häufigsten vorkommen, bei denen der vereinigte Stamm der Digitalis communis II. und III. entweder in die Obliqua proximalis (6 unter 18 Fällen) oder in die Digitalis communis IV. einmündet (5). Ferner fand er einen Typus (die II. und III. je selbständig in die Obliqua proximalis), in 3 Fällen, welchen ich unter meinen Exemplaren nicht treffen konnte. Andererseits müssten meine Exemplare nach seinem Einteilungssystem im ganzen in 19 Typen eingeteilt werden, wobei die beiden häufigsten Typen von Hirota nur in 4 bzw. 6 Fällen anzutreffen sind, während der häufigste Typus in meinem Material (Einmündung der II. nach einzelner Aufnahme der III. und IV. in die Marginalis fibularis, 8 unter 38 Fällen) bei Hirota fehlte. Da eine solche Typeneinteilung mir fast sinnlos erschien, so unterscheide ich vorläufig 4 Typen mit 2 bzw. 3 Übergangstypen, je nachdem, wieviele Digitales communes in die Obliqua dorsalis oder in die Marginalis fibularis einfliessen :-

Typus A: Die Digitales communes II., III. und IV. fliessen alle in die Obliqua dorsalis ein, 5 mal.

Typus B: Die II. und III. in die Obliqua und die IV. in die Marginalis fibularis, 4 mal.

Typus C: Die II. allein in die Obliqua und die III. und IV. in die Marginalis, 8 mal. 
Typus D: Die II., III. und IV. alle in die Marginalis, 17 mal. Typus BC und CD: Die III. bzw. IV. gegabelt in die benachbarten Venenstämmchen, je 2 mal.

Alles in allem wird die Anordnung der Venenstämmchen auf dem Fussrücken von Macacus vielleicht ungefähr folgendermassen typisiert werden:-Die Marginalis tibialis stellt die Fortsetzung der Tibialis hallucis dar, und die Marginalis fibularis die der Fibularis digiti V. Die Digitalis communis I. teilt sich in ein Crus tibiale und Crus fibulare; das erstere verbindet sich mit der Tibialis hallucis, während das letztere mit der Marginalis fibularis den Anfangsteil der Saphena parva liefert. Die Digitalis communis II. fliesst entweder in die Obliqua dorsalis oder in die Marginalis fibularis ein; die III. entweder in die II. oder in die IV.,

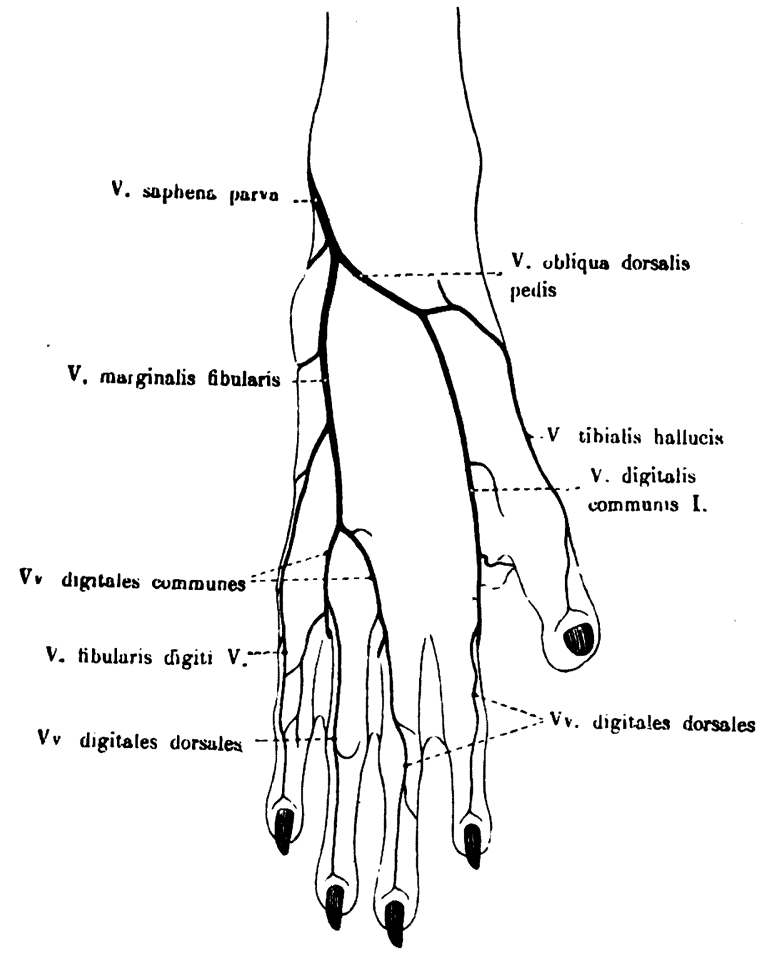

Fig. 2.

Die Hautvenen am Fussrücken von Semnopithecus entellus. und die IV. entweder in die II. oder in die Marginalis fibularis.

An der Plantarseite jeder Zehe findet sich ein langgestrecktes Hautvenennetz, welches an der Zehenbasis je eine Interdigitalis oder besser Digitalis plantaris über die tibiale bzw. fibulare Seite der Grundphalanx zur Digitalis dorsalis der betreffenden Zehe schickt und sich andererseits proximalwärts mit dem feinmaschigen Rete venosum plantare an der Fussohle zusammeuhängt. Das letztere verbindet sich durch mehrere $Z_{\text {weige }}$ über die beiden Fussränder mit den beiden Marginales, wobei der Blutstrom hauptsächlich proximalfibularwärts gerichtet ist.

So sammeln sich die Hautvenen des Fusses von Macacus vorwiegend proximal-fibularwärts in die Marginalis fibularis, welche weiter proximal- 
wärts in die Saphena parva übergeht. Nur die Venen der Grosszehe und des tibialen Fussrandes fliessen in die Marginalis tibialis ein, welche offenbar dem Anfangsteil der Saphena magna der höheren Primaten entspricht, deren phylogenetische Vorstufe gerade hier bei Macacus zu suchen ist.

Bei Semnopithecus und bei den von mir untersuchten 4 Platyrrhinenarten verhalten sich die Hautvenen des Fusses etwas anders. Beim ersteren fehlte die Marginalis tibialis, indem die Tibialis hallucis sämtlich durch die dem Crus tibialis der Digitalis communis I. entsprechenden Vene sich in die Obliqua dorsalis fortsetzte. Die Digitalis communis II. fehlte auch, und der aus der III. und IV. entstandene Venenstamm mündete rechts in die Marginalis fibularis, links dagegen in die Obliqua dorsalis ein. Die Digitalis communis III. und IV. empfingen rechts je die Digitalis dorsalis der 3. und der 4. Zehe; links

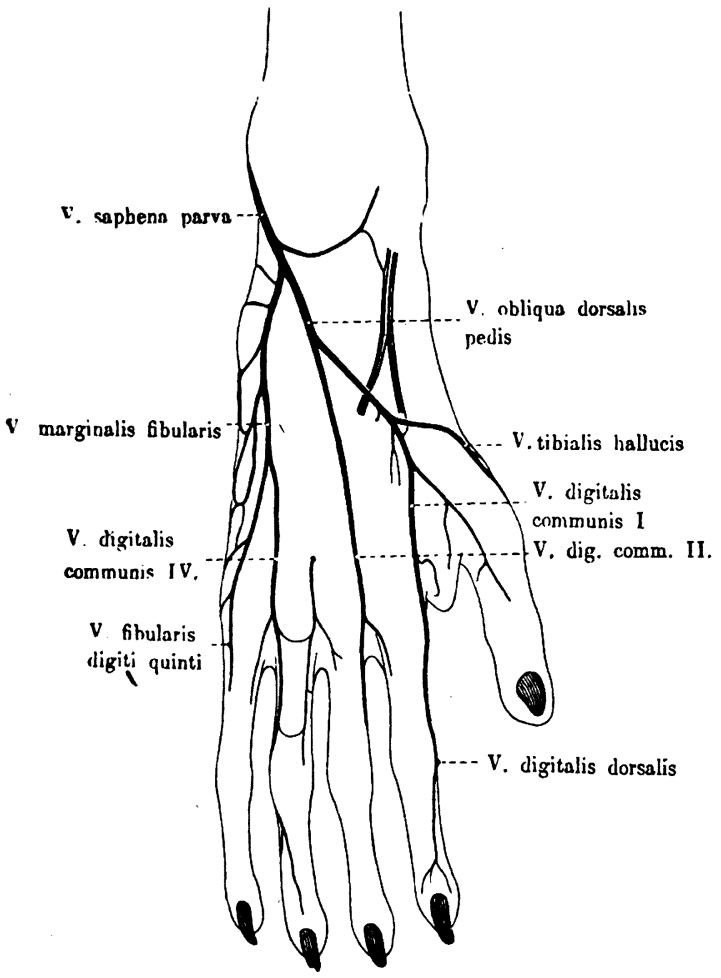

A.

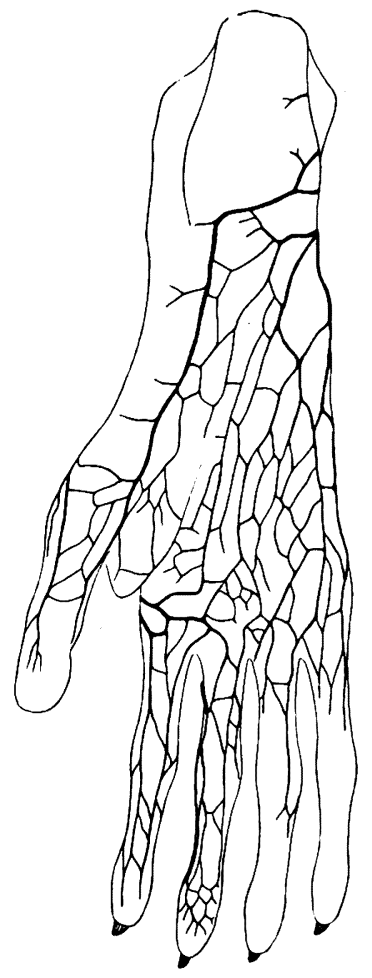

B.

Fig. 3.

Die Hautvenen am Fussrücken (A) und an der Fussohle (B) von Cebus caputinus. 
entstand die III. dagegen aus dem Zusammenfluss der von den entgegengesetzten Rändern der 3. und 4. Zehe kommenden Venenstämmchen, während die IV. hauptsächlich die Fortsetzung der Interdigitalis s. Digitalis plantaris der 4 . Zehe darstellte.

Bei den Platyrrhinen fehlte ebenfalls die Marginalis tibialis, und die Tibialis hallucis verhielt sich gerade so wie bei Semnopithecus. Die Digitalis communis II. fehlte bei Lagothrix wie bei Semuopithecus, mündete bei Hapale selbständig, bei Mystax nach Aufnahme der III. in die I., bei Cebus dagegen selbständig in die Obliqua dorsalis ein. Die Digitalis communis III. war bei allen Exemplaren sehr kurz und mündete bei Mystax gleich in die II., bei Hapale, Lagothrix und Cebus ebenfalls gleich in die IV. ein, welche bei allen ohne Ausnahme in die Marginalis fibularis aufgenommen wurde.

An der Plantarseite des Fusses verhielten sich die Hautvenen gerade so wie bei Macacus; ihr Blutstrom richtete sich proximal-fibularwärts und mündete durch mehrere Verbindungen in die Marginalis fibularis ein.

So scheint bei den Platyrrhinen und vielleicht auch bei den Semnipitheciden spezifisch zu sein, dass die Marginalis tibialis fehlt und demgemäss das erste phylogenetische Auftreten der Saphena magna noch ausbleibt, indem die sämtlichen Hautvenen des Fusses in die Obliqua dorsalis und in die Marginalis fibularis einfliessen, welche beide zusammen die Saphena parva bilden.

Vergleichen wir endlich die Venen des Fusses und der Hand der Affen miteinander, so stossen wir hier auf einen recht interessanten Kontrast. Die Venen des Handrückens sammeln sich, wie ihrerseits erörtert, hauptsächlich daumen- bzw. radialwärts, um zusammen die Cephalica zu bilden. Die Venen des Kleinfingers und des ulnaren Randes der Hand stellen dagegen ein selbständiges Stämmchen, Marginalis ulnaris, dar, welches sich am Processus styloides ulnae in 2 Schenkel teilt; der dorsale Schenkel, Obliqua dorsalis, fliesst auf der Rückenseite der Handwurzel proximal-radialwärts und mündet in die Cephalica ein, während der volare Schenkel, Obliqua volaris, nach Aufnahme der Venen aus dem Rete volare manus auf der volaren Seite des distalen Teils des Vorderarmes proximal-radialwärts verläuft und ebenfalls in die Cephalica einmündet. Die Cephalica ist also der einzige Venenstamm, welcher die Hautvenen von der Hand empfängt. Eine genügend starke Basilica, wie Hirota beschreibt, wurde niemals an meinen Exemplaren beobachtet, wenn auch der volare Schenkel der Marginalis ulnaris zweifellos die erste phylogenetische Andeutung der genannten Venen dokumentiert. 
Am Fuss ist der Zustand gerade umgekehrt. Die Hautvenen fliessen vorwiegend fibularwärts und bilden zusammen die Saphena parva. Die Saphena magna fehlt, welche nach Hirota erst bei Orang-Utan in voller Entwicklung gefunden wird; ihre phylogenetische Abstammung ist aber sicher in der Marginalis tibialis von Macacus zu suchen. Und diese Gegensätzlichkeit im Venensystem der Hand und des Fusses scheint m. E. hauptsächlich darauf zu beruhen, dass die Vorder- und Hinterextremität in der Phylogenese entgegengesetzte Entwicklungsbahn einschlugen, was bekanntlich besonders deutlich im Skelettsystem seine Ausprägung findet.

\section{Resumo en Esperanto.}

Pri la haŭtvejnoj de piedo ce simioj.

Materialo: 19 ekzempleroj de Macacus, ciu 1 de Semnopithecus entellus, Hapale jacchus, Mystax rosalia, Lagothrix lagotrica kaj Cebus capucinus.

Sur la dorsa surfaco de ciu fingro kuras ordinare nur unu veinotrunketo. Ĉe la 1. kaj 5. fingro ĝi faras V. tibialis hallucis kaj V. fibularis digiti quinti, kiuj daŭrigas sin proksimen en V. marginalis tibialis kaj V. marginalis fibularis. Ĉe ceteraj fingroj g $\mathrm{i}$ faras $V$. digitalis dorsalis, kaj daŭrigas sin post akcepto de V. digitalis plantaris el la vejuoreto ce planda surfaco de responda fingro en $\mathrm{V}$. digitalis communis. V. digitalis communis I. dividas sin en 2 brançojn: La tibiala branĉo kunfluas kun V. tibialis hallucis kaj faras V. marginalis tibialis, kiu kuras trans la tibian maleolon proksimen kaj enfluas en $V$. saphena parva. La fibulara branĉo, V. obliqua dorsalis, kuras sur piedodorso fibularproksimen kaj kunmetas kun V. marginalis fibularis la komencan parton de V. saphena parva. Vv. digitales communes II., III. kaj IV. enfluas diversmàniere a $\bar{u}$ en $V$. obliqua dorsalis ă̆ en $V$. marginalis fibularis. Ce plando la haŭtovejnoj faras delikatan reton; la vejnoj elfluantaj el la reto enfluas cefe en $V$. marginalis fibularis.

Ĉe Semnopithecus kaj ce 4 de mi esploritaj platirinoj mankis V. marginalis tibialis kaj la haŭtvejnoj de piedo kolektigisis çiuj en $\mathrm{V}$. obliqua dorsalis kaj $\mathrm{V}$. marginalis fibularis, por kummeti la komencan parton de V. saphena parva.

V. saphena magna, kies filogenezan antaŭiranton faras V. marginalis tibialis, mankis ce ĉiuj simiekzempleroj esploritaj. 